\title{
Osteochondral Lesion in Diffuse Pigmented Villonodular Synovitis of the Knee
}

\author{
Anshu Shekhar, $\mathrm{MS}^{1}$, Savneet Singh, $\mathrm{MS}^{1}$, Shantanu Sudhakar Patil, $\mathrm{MS}^{2}$, and \\ Sachin Ramchandra Tapasvi, MS, FRCS ${ }^{1}$ \\ ${ }^{1}$ Department of Orthopaedic Surgery, The Orthopaedic Speciality Clinic, Pune; ${ }^{2}$ Department of Orthopaedic Surgery, SRM Medical College Hospital and Research \\ Centre, Tamil Nadu, India
}

\begin{abstract}
Pigmented villonodular synovitis (PVNS) is a rare benign condition that is locally aggressive and may destructively invade the surrounding soft tissues and bone causing functional loss of the joint and the limb. The knee is the most affected joint (range, 28\% to $70 \%$ ) but involvement of the bone is not a common feature seen at this site. We present a rare case of diffuse PVNS of the knee associated with subchondral cyst of the lateral femoral condyle. This posed a diagnostic dilemma because of bone invasion. The radiological image of synovitis was pathognomonic of PVNS but etiology of the osteolytic lesion was confirmed only on histopathology. The large osteochondral defect was eventually managed in a staged manner with bone grafting and osteochondral autograft transfer.
\end{abstract}

Keywords: Knee, Cartilage, Synovitis, Pigmented villonodular

Pigmented villonodular synovitis (PVNS) is a rare, benign, but potentially recurrent condition with an estimated incidence of 1.8 per million. It is characterized by synovial proliferation and hemosiderin deposition inside the joints, tendon sheaths, and bursae. It is a locally aggressive lesion that may invade and destroy surrounding soft tissue and bone, resulting in functional deterioration of the joint and the extremity. It may involve any synovial joint, but large joints are frequently affected. The knee is the most affected site (range, 28\% to 70\%), but cases in the hip, ankle, shoulder, and elbow are often observed ${ }^{1)}$. The disease presents in two forms: when the entire synovium of a joint is affected, the condition is referred to as diffuse PVNS and when a single

Received February 5, 2018; Revised (1st) August 1, 2018;

(2nd) September 4, 2018; Accepted October 2, 2018

Correspondence to: Anshu Shekhar, MS

Department of Orthopaedic Surgery, The Orthopaedic Speciality Clinic, 16 Status Chambers, 1221/A Wrangler Paranjpe Road, Pune 411004, India

Tel: +91-91-4605-0657

E-mail: dr.anshushekhar@gmail.com

This is an Open Access article distributed under the terms of the Creative Commons Attribution Non-Commercial License (http://creativecommons.org/licenses/by-nc/4.0/) which permits unrestricted non-commercial use, distribution, and reproduction in any medium, provided the original work is properly cited. discrete mass is present in the synovium, it is known as localized PVNS. Microscopically, PVNS is characterized by the presence of lipid-laden macrophages, multinucleated giant cells, hemosiderin deposits, and proliferation of fibroblasts and stromal cells. Both localized and diffuse variants have similar histological appearance.

It is extremely unusual for the PVNS mass to invade the bone or to be present in an intra-osseous location, especially in the knee joint. We present a rare presentation of diffuse PVNS of the knee associated with subchondral cyst of the lateral femoral condyle (LFC) and chondral defect and its management.

\section{Case Report}

A 35-year-old male presented with a seven-month history of pain, swelling and locking in his left knee joint. The swelling was gradually progressive and caused difficulty in walking and squatting. On examination, there was effusion and synovial thickening with tenderness at the lateral joint line but no local warmth. The range of motion was full without pain. McMurray test was positive and signs of joint instability were negative. There was no involvement of any other joint and personal and family history were unremarkable. Plain radiographs of the right knee revealed 
an osteolytic area in the LFC. A 3 Tesla magnetic resonance imaging (MRI) scan (Ingenia, Philips, Netherlands) showed abnormal intra-articular soft tissues in the posterior aspect of the intercondylar region adjacent to the posterior cruciate ligament and the lateral part of the suprapatellar pouch which were hyperintense on proton density fat saturated images with multiple areas of gradient blooming on gradient recalled echo (GRE) images and significant post contrast enhancement. There was the presence of a subchondral geode measuring $18 \times 18 \times 25 \mathrm{~mm}$ in the posterior aspect of the LFC with surrounding marrow edema and adjacent full thickness chondral defect measuring $6 \times 11 \mathrm{~mm}$ (Fig. 1). A 3 phase $99 \mathrm{~m}$ Technetium-methyl diphosphonate (Tc-MDP) bone scan was done wherein the flow, blood pool and delayed static images showed an increased uptake in the left knee region only, suggestive of low grade inflammatory pathology and no multifocal involvement.

The preliminary differential diagnoses included PVNS, giant cell tumor, reactive synovitis, low grade infection and synovial chondromatosis based on a review of clinical and radiological features of synovitis with subchondral geode ${ }^{2)}$. The patient was counselled for arthroscopic excision of the lesion considering a provisional diagnosis of PVNS. Under spinal anaesthesia, arthroscopic excision of the entire diseased synovium was performed using a radiofrequency device and a rotatory shaver utilizing anterolateral, anteromedial, superomedial, superolateral, posterolateral and posteromedial portals (Fig. 2). The medial meniscus was found to have a horizontal cleavage tear which was repaired using two Ultra Fast-Fix implants (Smith and Nephew, Andover, MA, USA). There was a grade IV International Cartilage Repair Society chondral defect over the weight bearing area of the LFC. Probing of the subchondral bone at the defect caused a breakdown of the floor of the defect, revealing the intra-osseous cyst with haemorrhagic fluid and tissue. The cyst was thoroughly curetted and its walls were burred till fresh bleeding bone was
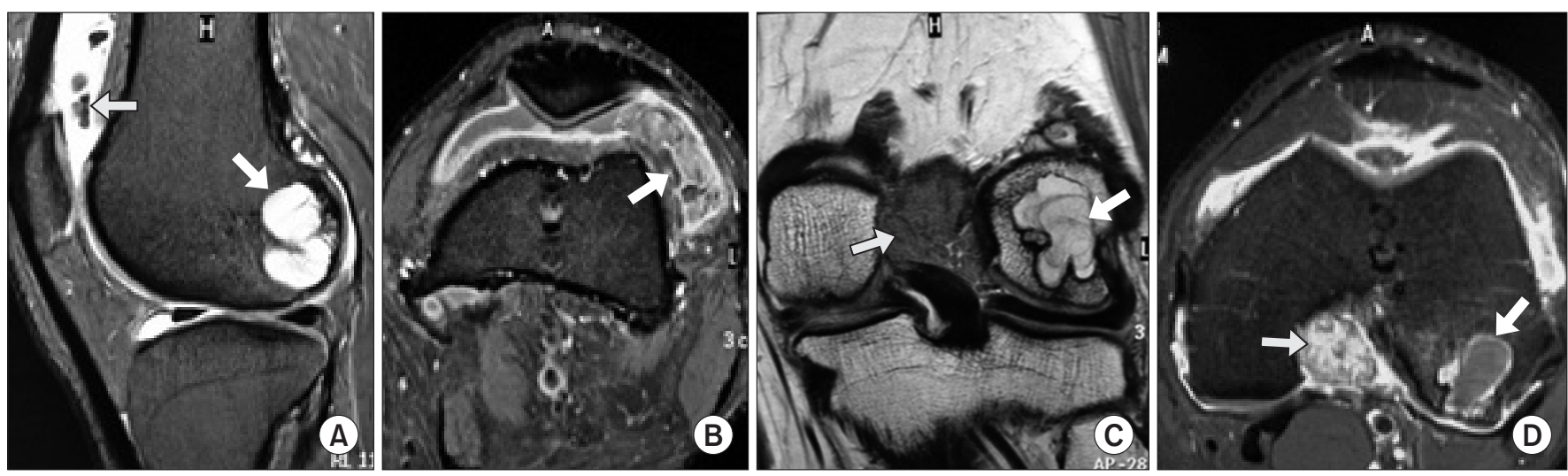

Fig. 1. Preoperative magnetic resonance imaging. (A) Proton density sagittal image showing a bony lesion in the posterior aspect of the lateral femoral condyle (thick arrow) and suprapatellar pouch (thin arrow). (B) In axial section, the synovial lesion in lateral aspect of suprapatellar pouch (arrow) demonstrates contrast enhancement. (C) T1-weighted coronal image showing the bone cyst localized in the posterior lateral femoral condyle (thick arrow) and synovial lesion in the intercondylar area superior to the posterior cruciate ligament (thin arrow). (D) Proton density axial image showing the extent of lesion in the bone (thick arrow) and synovium in the intercondylar area (thin arrow).
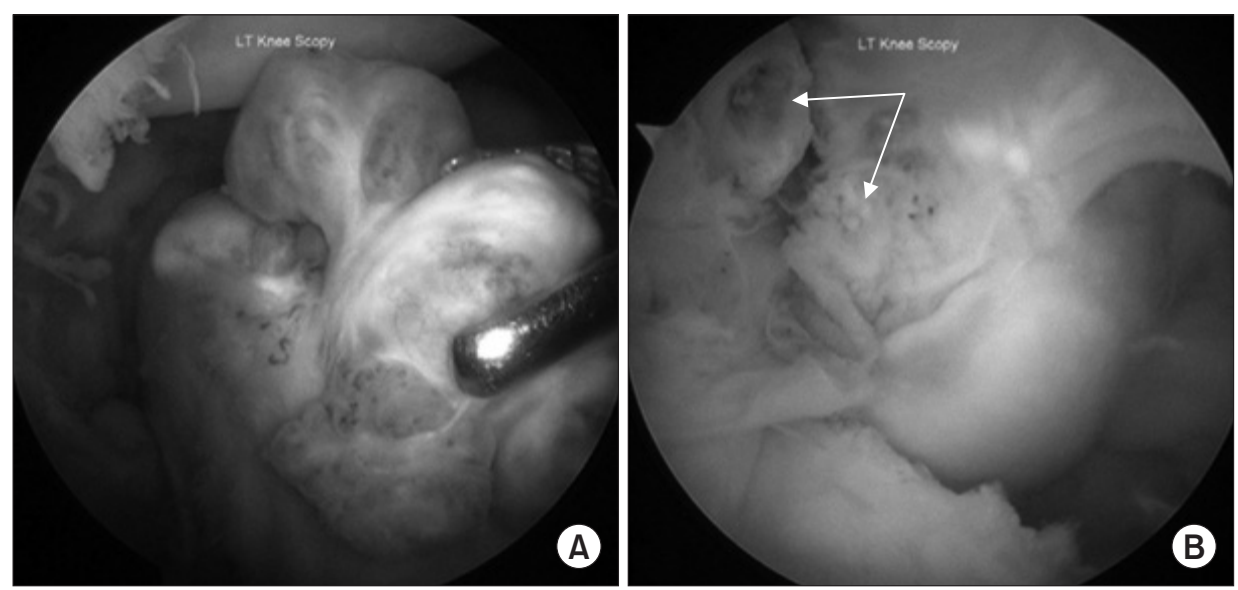

Fig. 2. Arthroscopic view of synovial lesions. (A) The synovial tumors are seen in the lateral suprapatellar area while viewing through the superolateral portal and the grasper introduced through the superomedial portal. (B) The synovial lesions are seen in the posterior compartment behind the posterior cruciate ligament (arrow) while viewing from the posterolateral portal. 

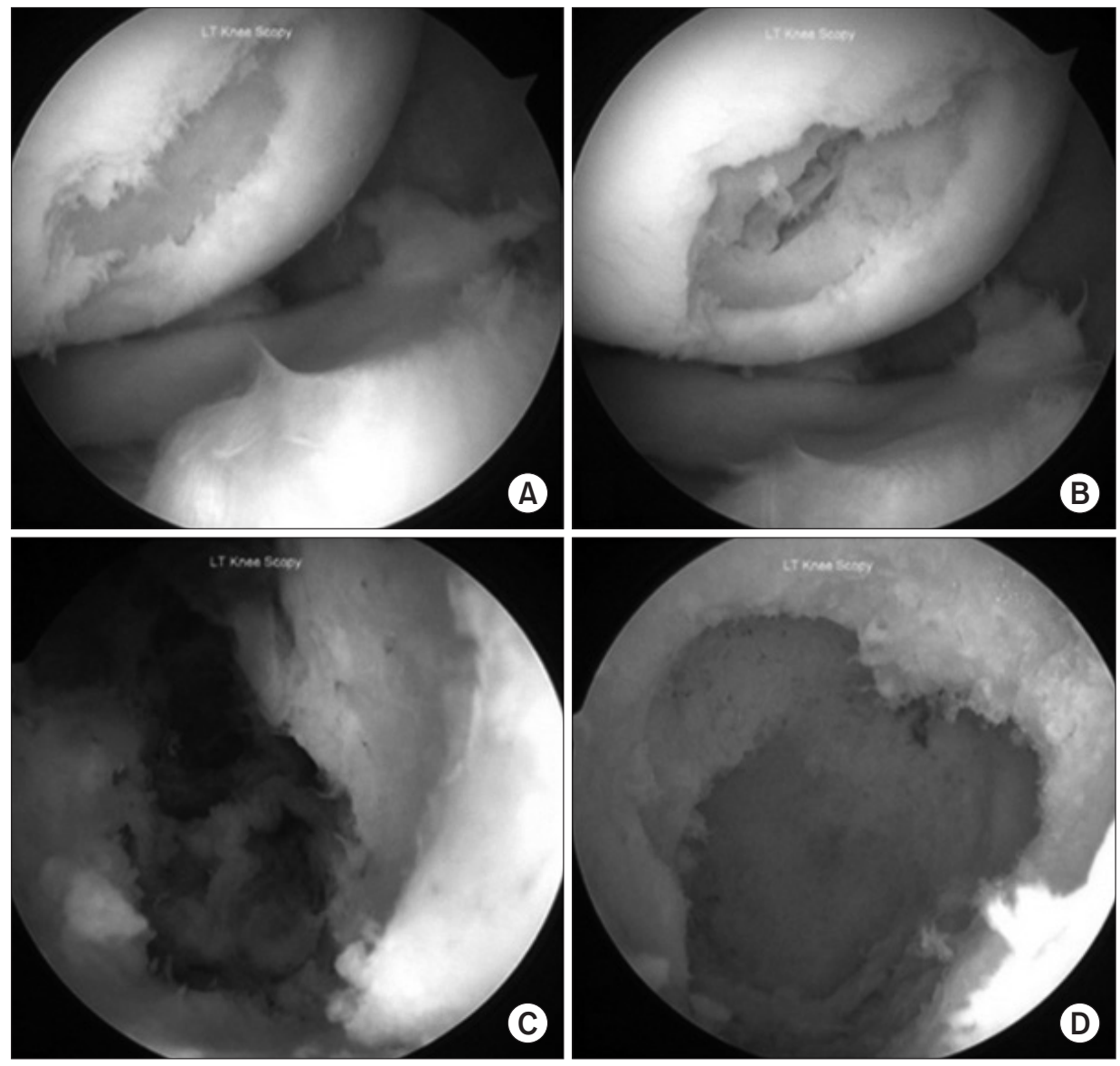

Fig. 3. Arthroscopic view of the osteochondral lesion. (A) Full thickness cartilage defect over the weight bearing portion of lateral femoral condyle as seen from the anteromedial portal. (B) Breakdown of the subchondral bone upon probing the base of the cartilage defect. (C) The interior of the bone cyst as seen by the arthroscope inserted from the anterolateral portal revealed hemorrhagic tissue within the cavity. (D) The interior of the cyst as seen after complete curettage of the cavity.

seen all around. At the end of the procedure, the chondral defect measured $10 \times 10 \mathrm{~mm}$ and depth of the defect was $28 \mathrm{~mm}$ (Fig. 3). The tissues from the synovectomy and cyst curettage were sent for histopathology separately. Considering the large size of bone defect and unknown nature of the lesion, a definitive chondral restorative procedure was not performed simultaneously and planned for a second stage. Postoperatively, the patient was kept non-weight bearing and allowed full range of motion. Quadriceps strengthening exercises and ice therapy were started.

Histopathology revealed synovial cell hyperplasia, multinucleated giant cells, macrophages and hemosiderin deposit from both specimens (synovium and intra-osseous lesion). The patient was explained about the need for management of the LFC cartilage defect and underlying bone defect in the weight bearing zone. Two months after the index procedure, he was posted for the second surgery. Under spinal anaesthesia, the defect was approached through a lateral parapatellar arthrotomy. The walls of the cavity were curetted again. The cavity was filled with synthetic bone graft substitute granules containing $\beta$-tricalcium phosphate (ChronOS; DePuy Synthes, West Chester, PA, USA) and impacted. Cancellous bone graft from the ipsilateral fibular head was then impacted over this with the alignment rod provided in the OATS set (Arthrex, Naples, FL, USA) to obtain a cylindrical defect. A 10-mm diameter, 25-mm long osteochondral plug was harvested from the proximal lateral LFC margin which is nonarticular with the patella and inserted into the defect to obtain a press-fit (Fig. 4). Postoperatively, the patient was kept non-weight bearing for four weeks, followed by partial weight bearing for two weeks, and then was allowed full weight bearing.

At eighteen months of follow-up, the patient was asymptomatic with no signs of recurrence. A 3 Tesla MRI was performed which showed no evidence of recurrent or residual synovial disease. The bone defect had completely consolidated and the implanted osteochondral autograft cartilage was well incorporated (Fig. 5).

\section{Discussion}

PVNS generally affects patients in the third and fourth decades of life with pain and swelling of the joint being commonly presenting symptoms. MRI is the imaging of choice for diagnosis as it is non-invasive and highly accurate for assessing the extent of the disease and distinguishing between the diffuse and localized 

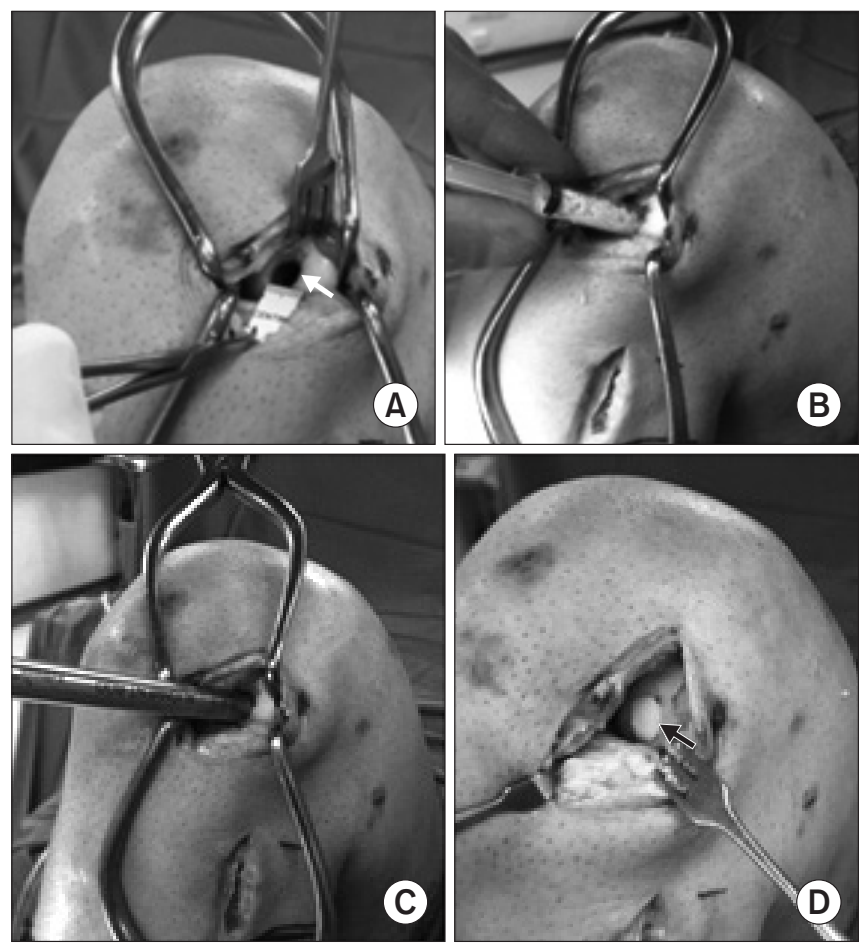

Fig. 4. Second stage surgery for management of the osteochondral defect. (A) The chondral defect was $10 \mathrm{~mm}$ (white arrow) as seen through the lateral parapatellar arthrotomy. (B) The bone cavity being filled with $\beta$-tricalcium phosphate granules using a $10 \mathrm{cc}$ syringe to prevent spillage in the joint. (C) The alignment rod from OATS set (Arthrex) being used for impaction of the graft into the defect. (D) Insertion of a $10 \mathrm{~mm}$ osteochondral plug (black arrow) into the defect.

variants. Our patient had masses in the synovium in the posterior aspect of the intercondylar notch and suprapatellar pouch which were hyperintense on proton density sequence with gradient blooming on GRE images and significant post contrast enhancement. These findings are very typical of PVNS but the presence of large subchondral geode is not. In one third of diffuse PVNS, mainly in a chronic setting, bony changes mainly in the form of margin erosions and cysts surrounded by thin sclerosis are seen ${ }^{3)}$. McMaster $^{4)}$ presented six cases of PVNS with bone invasion. He was perhaps the first to report invasion of bone in the knee joint in the English literature. Pantazopoulos et al. ${ }^{5}$ presented seven cases of PVNS with bone lesions. The hip joint was affected in four patients, and in the remaining three patients, the knee, shoulder and ankle were involved. Radiographically, the most common finding was bone cysts situated at some distance from the articular surfaces, usually well-defined. Histological examination revealed villous hypertrophy of the synovial membrane, and the involved bone showed thinner trabeculae and cysts, containing similar synovial tissue on histopathological examination ${ }^{5}$. These findings are similar to our case.
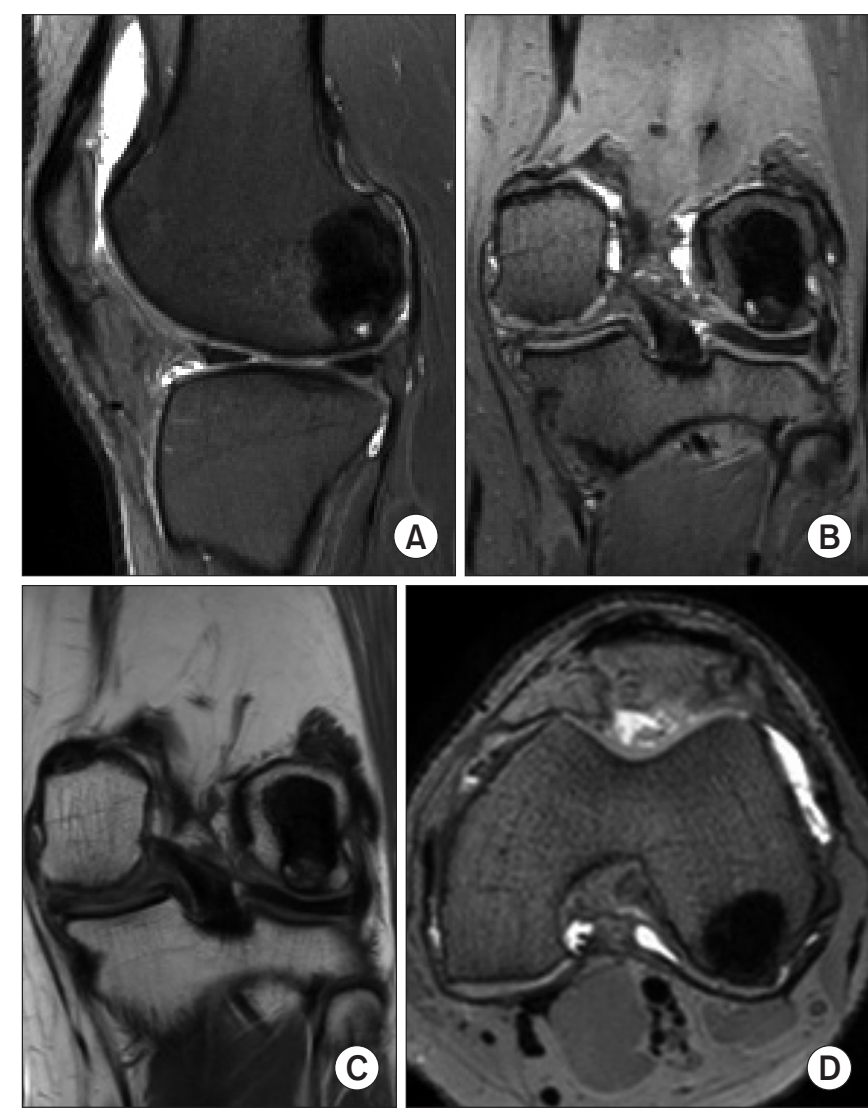

Fig. 5. Postoperative magnetic resonance imaging (MRI) at the final follow-up: the bone defect has completely consolidated and the implanted osteochondral plug is well incorporated with no surrounding edema as seen on the proton density sagittal (A), proton density coronal (B), T1weighted coronal (C) and proton density axial (D) images.

Being aware of the possible presence of these bone changes in PVNS obviates confusion with other conditions that can present with a large epiphyseal lytic lesion like giant cell tumor, reactive synovitis, low grade infection and synovial chondromatosis. There is one case of PVNS with bone changes reported in the literature, which was misdiagnosed as malignant synovioma and hind-quarter amputation was performed ${ }^{6}$. In another case of PVNS with bone changes affecting the hip joint, the synovial proliferation had a retroperitoneal extension simulating an abdominal tumour ${ }^{7}$. Pathogenesis of these intra-osseous lesions is not known. Chung and Janes ${ }^{8}$ postulated that exuberant villonodular tissue and effusion of the joint cause high intra-articular pressure. This in turn results in small areas of osteoporosis near the joint where the bone cysts are developed. The thus-formed cysts are finally invaded by the hypertrophic synovium of the joint through fractures of the cystic walls. According to McMaster ${ }^{4)}$, the cysts are created by the extension of villonodular tissue into the bone through the chondro-osseous area at the articular margin. 
Scott ${ }^{9)}$ postulated that the invasion of bone by the hypertrophic synovium takes place through the vascular foramina along with epiphyseal vessels. As no macroscopic breach was detected, it is speculative to comment as to how the lesion might have invaded the bone in this case.

Treatment options for diffuse PVNS can be arthroscopic excision, open synovectomy, combined arthroscopic and open synovectomy, with or without adjuvant radiotherapy. In a recent literature review of diffuse PVNS by Auregan et al. ${ }^{10)}$, no difference was found in terms of local recurrence following open synovectomy (22.6\%) or arthroscopic synovectomy (16.1\%), but complication rates were much higher following open synovectomy. No adjuvant treatment was given to our patient as complete arthroscopic synovectomy was possible in this case. The bone lesion was managed successfully by grafting with synthetic bone substitute and autologous cancellous graft. Management of the chondral defect by autologous osteochondral transfer was successfully done to restore the cartilage status. Thus, joint preservation is imperative where the bone involvement is focal or localized. The absence of recurrence and excellent functional outcome were due to the optimal management of this unusual case.

PVNS is a rare condition affecting the joints and presence of a bone lesion in association with it is even rarer, especially in the knee joint. We came across this case where a knee diffuse PVNS had invaded the bone, thus causing a conundrum in diagnosis. Arthroscopic synovectomy to remove the entire diseased synovium was performed along with curettage of the cyst, which resulted in a large osteochondral defect. This defect was treated with bone grafting and implantation of an osteochondral autograft plug in the second stage of the surgery with successful outcome.

\section{Conflict of Interest}

No potential conflict of interest relevant to this article was reported.

\section{References}

1. Deitcher SR, Carman TL. Deep venous thrombosis and pulmonary embolism. Curr Treat Options Cardiovasc Med. 2002;4:223-38.

2. Turan A, Celtikci P, Tufan A, Ozturk MA. Basic radiological assessment of synovial diseases: a pictorial essay. Eur J Rheumatol. 2017;4:166-74.

3. Temponi EF, Barros AAG, Paganini VO, Barbosa VAK, Badet R, Carvalho Junior LH. Diffuse pigmented villonodular synovitis in knee joint: diagnosis and treatment. Rev Bras Ortop. 2017;52:450-7.

4. McMaster PE. Pigmented villonodular synovitis with invasion of bone: report of six cases. J Bone Joint Surg Am. 1960; 42:1170-83.

5. Pantazopoulos T, Stavrou Z, Stamos C, Kehayas G, Hartofilakidis-Garofalidis G. Bone lesions in pigmented villonodular synovitis. Acta Orthop Scand. 1975;46:579-92.

6. Byers PD, Cotton RE, Deacon OW, Lowy M, Newman PH, Sissons HA, Thomson AD. The diagnosis and treatment of pigmented villonodular synovitis. J Bone Joint Surg Br. 1968; 50:290-305.

7. Carr CR, Berley FV, Davis WC. Pigmented villonodular synovitis of the hip joint; a case report. J Bone Joint Surg Am. 1954;36:1007-13.

8. Chung SM, Janes JM. Diffuse pigmented villonodular synovitis of the hip joint: review of the literature and report of four cases. J Bone Joint Surg Am. 1965;47:293-303.

9. Scott PM. Bone lesions in pigmented villonodular synovitis. J Bone Joint Surg Br. 1968;50:306-11.

10. Auregan JC, Klouche S, Bohu Y, Lefevre N, Herman S, Hardy P. Treatment of pigmented villonodular synovitis of the knee. Arthroscopy. 2014;30:1327-41. 